\title{
Efficacy of Long Term Indomethacin Therapy in Prolonging Pregnancy After Fetoscopic Laser Surgery for Twin-to-Twin Transfusion Syndrome: a Collaborative Cohort Study
}

\author{
Hiba Mustafa ${ }^{1}$, Eyal Krispin ${ }^{2}$, Hooman Tadbiri ${ }^{1}$, Jimmy Espinoza ${ }^{3}$, Alireza Shamshirsaz ${ }^{2}$, \\ Ahmed Nassr ${ }^{2}$, Roopali Donepudi ${ }^{3}$, Michael Belfort (FETAL MEDICINE ISSUE \\ EDITOR ${ }^{2}$, Magdalena Sanz Cortes ${ }^{4}$, Nicole Pedersen ${ }^{1}$, Christopher Harman ${ }^{5}$, and Ozhan \\ $\operatorname{Turan}^{5}$ \\ ${ }^{1}$ University of Maryland Baltimore \\ ${ }^{2}$ Baylor College of Medicine \\ ${ }^{3}$ Baylor College of Medicine and Texas Childrens Hospital Pavilion for Women, Houston, \\ TX, United States \\ ${ }^{4}$ BAYLOR COLLEGE OF MEDICINE, TEXAS \\ ${ }^{5}$ University of Maryland School of Medicine
}

July 10, 2021

\begin{abstract}
Objective: To evaluate the efficacy of long-term indomethacin therapy (LIT) in prolonging pregnancy and reducing spontaneous preterm birth (PTB) in patients undergoing fetoscopic laser surgery (FLS) for the management of twin-to-twin transfusion syndrome (TTTS). Design: Retrospective cohort study of prospectively collected data. Setting: Collaborative multicenter study Population: 557 consecutive TTTS cases that underwent FLS Methods: LIT was defined as indomethacin use for at least 48 hours. Log-binomial regression was used to estimate the relative risk (RR) of PTB in LIT compared to non-LIT group. Cox regression was used to evaluate the association between LIT use and FLS-to-delivery survival. Main outcome measures: gestational age (GA) at delivery Results: Among the 411 pregnancies included, a total of 180 patients (43.8\%) received LIT after FLS and 231 patients (56.2\%) did not. Median GA at fetal intervention did not differ between groups (20.4 weeks). Median GA at delivery was significantly higher in the LIT group (33.6 weeks) compared to the non-LIT group (31.1weeks), $\mathrm{p}<0.001$. FLS-to-delivery interval was significantly longer in the LIT group $(\mathrm{P}<0.001)$. The risk of PTB prior to $34,32,28$, and 26 weeks gestation were all significantly lower in the LIT group compared to the non-LIT group $(\mathrm{RR}=0.69,0.51,0.37$, and 0.18 , respectively). The number needed to treat (NNT) with LIT to prevent one PTB $<32$ weeks gestation was 4 , and to prevent one PTB $<34$ weeks was 5. Conclusion: Long-term indomethacin after FLS for TTTS was found to be associated with prolongation of pregnancy and reducing the risk for PTB.
\end{abstract}

Short title: Long term indomethacin in TTTS treated with FLS

Hiba J. Mustafa ${ }^{1}$, MD; Eyal Krispin ${ }^{2}$, MD; Hooman Tadbiri ${ }^{1}$, MD MPH; Jimmy Espinoza ${ }^{2}$, MD; Alireza A. Shamshirsaz ${ }^{2}$, MD; Ahmed A. Nassr ${ }^{2}$, MD; Roopali Donepudi ${ }^{2}$, MD; Michael A. Belfort ${ }^{2}$, MD PhD; Magdalena Sanz Cortes ${ }^{2}$, MD PhD; Nicole Pederson ${ }^{1}$, BSN, RN, SCNII; Christopher Harman ${ }^{1}$, MD; Ozhan M. Turan ${ }^{1}, \mathrm{MD} \mathrm{PhD}$

${ }^{1}$ Department of Obstetrics, Gynecology \& Reproductive Sciences, University of Maryland School of Medicine, Baltimore, MD. 
${ }^{2}$ Department of Obstetrics and Gynecology, Baylor College of Medicine and Texas Children's Fetal Center, Houston, Texas, USA

Address correspondence to:

Ozhan M. Turan, MD PhD

Department of Obstetrics, Gynecology \& Reproductive Sciences, Division of Maternal-Fetal Medicine, University of Maryland School of Medicine, Baltimore, MD.

Email: oturan@som.umaryland.edu

\section{Abstract}

Objective: To evaluate the efficacy of long-term indomethacin therapy (LIT) in prolonging pregnancy and reducing spontaneous preterm birth (PTB) in patients undergoing fetoscopic laser surgery (FLS) for the management of twin-to-twin transfusion syndrome (TTTS).

Design: Retrospective cohort study of prospectively collected data.

Setting: Collaborative multicenter study

Population: 557 consecutive TTTS cases that underwent FLS

Methods: LIT was defined as indomethacin use for at least 48 hours. Log-binomial regression was used to estimate the relative risk (RR) of PTB in LIT compared to non-LIT group. Cox regression was used to evaluate the association between LIT use and FLS-to-delivery survival.

Main outcome measures : gestational age (GA) at delivery

Results: Among the 411 pregnancies included, a total of 180 patients (43.8\%) received LIT after FLS and 231 patients (56.2\%) did not. Median GA at fetal intervention did not differ between groups (20.4 weeks). Median GA at delivery was significantly higher in the LIT group (33.6 weeks) compared to the non-LIT group (31.1weeks), $\mathrm{p}<0.001$. FLS-to-delivery interval was significantly longer in the LIT group $(P<0.001)$. The risk of PTB prior to 34, 32, 28, and 26 weeks gestation were all significantly lower in the LIT group compared to the non-LIT group ( $\mathrm{RR}=0.69,0.51,0.37$, and 0.18 , respectively). The number needed to treat $(\mathrm{NNT})$ with LIT to prevent one $\mathrm{PTB}<32$ weeks gestation was 4 , and to prevent one $\mathrm{PTB}<34$ weeks was 5 .

Conclusion: Long-term indomethacin after FLS for TTTS was found to be associated with prolongation of pregnancy and reducing the risk for PTB.

\section{Tweetable abstract:}

Long term indomethacin used after fetoscopic laser surgery for twin-to-twin transfusion syndrome is effective in prolonging pregnancy and reducing the risk for preterm birth; especially extreme preterm birth.

Keywords: Indomethacin, long term indomethacin, fetoscopic laser, twin-to-twin transfusion, monochorionic diamniotic, twin

\section{Main text}

\section{Introduction}

Twin-to-twin transfusion syndrome (TTTS) affects approximately 10-15\% of monochorionic diamniotic twin pregnancies (MCDA). ${ }^{1}$ Fetoscopic laser surgery (FLS) has become the standard treatment for managing these pregnancies. ${ }^{2}$ Despite the apparent success of FLS, spontaneous preterm birth (sPTB) remains to be 
a major complication and a challenge that determines perinatal morbidity and survival, ${ }^{3,4}$ with a reported gestational age (GA) at delivery of 31 to 33 weeks of gestation. ${ }^{5,6} \mathrm{sPTB}$ accounts for almost $48 \%$ of these deliveries ${ }^{7}$ which has been largely related to preterm labor and preterm prelabor rupture of membranes (PPROM) with occurrence to as high as $53 \%$ in some reports. ${ }^{5,8,9}$ Up to date, effective PTB preventative measures in this population are lacking.

Indomethacin, a non-specific cyclooxygenase (COX) inhibitor, is a commonly used tocolytic agent. The three proposed mechanisms of action include COX blockage, uterine calcium efflux blockage, which reduces uterine resting tone, and the hydrophobic effect on cervical matrix metalloproteinases (MMP), which prevents further softening and shortening. ${ }^{10,11}$ Long-term indomethacin therapy (LIT) (>48 hours, and not beyond 32 weeks gestation) used under strict follow-up guidelines has shown effectiveness in stabilizing cervical length (CL) in dichorionic diamniotic twin (DCDA) pregnancies with a short cervix. ${ }^{12}$ The safety of LIT in the setting of the short cervix in singleton pregnancies has been demonstrated in a cohort study which showed no significant differences in fetal and neonatal complications compared to a matched control group. ${ }^{13}$

The hypothesis of our study was that LIT prolongs pregnancy and reduces the risk for sPTB in the setting of TTTS treated with FLS.

\section{Materials and Methods}

This was a retrospective cohort study of prospectively collected data of consecutive TTTS cases that underwent FLS at two fetal treatment centers between 2012 and 2018. The study was approved by the institutional review boards of both participating institutions (University of Maryland, Baltimore [UMB], MD and Baylor College of Medicine [BCM], Houston, TX). The study results are reported according to the Strengthening the Reporting of Observational Studies in Epidemiology (STROBE) guidelines for cohort studies. ${ }^{14}$

\section{Preoperative evaluation and management}

Patients referred for evaluation of TTTS, underwent comprehensive ultrasound examination, including biometry measurements with Doppler studies for the diagnosis and staging of TTTS based on the Quintero system, 15 performed by registered diagnostic medical sonographers supervised by experienced fetal medicine specialists. All cases with TTTS Stage II or higher were offered surgical intervention, as were Stage-I cases if they presented with a short cervix of less than $25 \mathrm{~mm}$ or symptomatic polyhydramnios. Patients undergoing FLS at UMB were started on the LIT protocol (see below in the LIT protocol section for details).

\section{Operative procedure}

FLS was performed using a single port fetoscope under either local anesthetic in conjunction with intravenous sedation or regional anesthesia. The entry technique used was either the Seldinger or sharp trocar method. The placental vascular equator was identified, and a diode laser used to ablate all intertwin vascular anastomoses using selective or sequential selective method followed by equatorial dichorionization 'Solomon technique' ${ }^{16}$ if technically feasible. When needed, amnioinfusion was performed to improve visualization. If amniodrainage was necessary, it was performed following the coagulation phase of the procedure.

\section{Postoperative evaluation and management}

Patients remained in the hospital for a minimum of 24 hours. Daily ultrasound exams were performed while patients remained in the hospital. After discharge, patients were either followed with weekly scans by the fetal center if feasible or returned to the referring provider with recommended follow-up care per institutional protocol. The decision for delivery was based on the referring physician's assessment. Information about pregnancy outcomes was collected prospectively either from the patients or the referring physicians as a part of follow-up care. 


\section{LIT protocol}

LIT was administered as a standard regimen using oral indomethacin of $100 \mathrm{mg}$ loading dose, then $50 \mathrm{mg}$ every 6 hours for up to 48 hours, followed by a maintenance dose of $25 \mathrm{mg}$ every 6 hours thereafter. The minimum duration was 48 hours and not beyond 32 weeks gestation. LIT ultrasound surveillance included weekly evaluation of the maximum vertical pocket (MVP) and Doppler flow studies (ductus arteriosus [DA], ductus venosus, and atrioventricular valves). These weekly scans were continued as long as patients were still using indomethacin. LIT was stopped if 1 . The patient was returned to the referring provider, where performing the weekly LIT ultrasound surveillance was not feasible, knowing that cervical length was stable. This category is referred to as "stable clinical status" throughout the paper, 2. LIT complications, including DA constriction, defined as the DA peak systolic velocity exceeded the $95^{\text {th }}$ percentile for GA, and/or the DA pulsatility index (PI) was $<5^{\text {th }}$ percentile for GA, or oligohydramnios defined as MVP of less than 2 $\mathrm{cm}, 3$. PPROM, and 4. Reaching 32 weeks gestation. ${ }^{13}$

\section{Study groups and outcome measures}

LIT in the setting of TTTS treated with FLS was used routinely after FLS in one of the participating institutions (UMB), while TTTS cases from the second institution (BCM) did not receive LIT. Exclusion criteria included triplet gestations, pregnancies that underwent cervical cerclage placement, postoperative complications that resulted in dual twin demise, obstetrical and medical indicated deliveries at less than 32 weeks gestation (did not encounter PTL or PPROM), and patients with missing records. Collected data included preoperative, operative, and postoperative variables, delivery information, and perinatal survival.

Preoperative variables included maternal characteristics (age, parity, race, previous PTB, BMI), TTTS stage using the Quintero criteria, CL (measured transvaginally), recipient's MVP, presence of anterior placenta, and presence of selective fetal growth restriction ( $\mathrm{sFGR}$ ). Operative variables included GA at FLS, size of trocar outer sheath in $\mathrm{mm}$, number of anastomoses ablated, whether or not complete dichorionization was performed, the occurrence of chorioamnion membrane separation (CAS), and septostomy (intertwin membrane disruption). Postoperative variables included GA at delivery, live births, and neonatal survival. The primary outcome measure was GA at delivery. Secondary outcome measures included FLS-to-delivery interval, live births, and neonatal survival.

\section{Statistical analysis}

Study data were deidentified and imported into the statistical software. Results are reported as mean \pm standard deviation (SD), median (interquartile range $[\mathrm{IQR}]$ ), or number (percentage). Normal distribution was determined by Shapiro-Wilk test. Categorical variables were compared using the Pearson's chi-square or Fisher's exact test. Continuous variables were compared using an unpaired $t$-test for normally distributed data or the Wilcoxon rank-sum test for not normally distributed data. Pearson correlation was used to assess linear correlation between two continuous variables.

Log-binomial regression was used to directly estimate relative risks (RRs) of PTB, very PTB, and extremely PTB. Significant variables in bivariate analysis were incorporated into a log-binominal regression model to determine which factors were independent risk factors of the primary outcome (PTB) and estimate adjusted RR. The number needed to treat (NNT) was calculated using pooled absolute risk reduction for PTD.

A secondary analysis was performed to evaluate FLS-to-delivery duration. Kaplan-Meier curve was used to visualize the FLS-to-delivery survival function for each group, and equality of survival functions between groups was tested using the log-rank test. Cox regression analysis was used to estimate hazard ratios (HRs) for FLS-to-delivery survival. Multivariate stepwise backward Cox regression was used to identify significant independent maternal characteristics, pre-operative variables, and operative variables that predict FLS-todelivery survival and estimate adjusted HR.

Trocar outer sheath diameter and total number of anastomoses were categorized into quartiles, age was categorized into $<35$ and [?]35 (advanced maternal age), BMI was categorized into $<25$ (underweight or 
normal), 25 - 30 (overweight), and [?]30 (obese), parity was categorized into 0,1 , and 2 or more, MVP was categorized into $<8$ (oligohydramnios or normal), $8-12$ (polyhydramnios), [?]12 (severe polyhydramnios), and GA at FLS was categorized into $<18$ weeks, $18-24$ weeks, and [?]24 weeks in log-binomial and Cox regression models to allow non-linear relationships. The $\mathrm{p}$ - value $<0.05$ was considered statistically significant. Statistical analysis was performed using the Stata 16 software. ${ }^{\mathbf{1 7}}$

\section{Results}

A total of 557 FLS procedures were performed during the study period. In this study, a total of 146 pregnancies were excluded. (Figure 1) The distribution of the excluded pregnancies was similar between the two centers (Table 1). After applying the exclusion criteria, 411 pregnancies were included in the final analysis; of those, 180 pregnancies received LIT (43.8\%) and 231 did not (56.2\%).

\section{Maternal characteristics and preoperative variables}

There were no statistically significant differences in maternal age, prior PTB, sFGR, CL $<25 \mathrm{~mm}$, presence of anterior placenta, and Quintero staging between the two groups (Table 2). Preoperative significant differences between groups were race, of which $69.3 \%$ were white in the LIT group vs. $54.5 \%$ in the non-LIT group $(\mathrm{p}<0.001)$, BMI with the median of 23.95 (IQR: 21 - 29.4) in the LIT group vs. 28.2 (IQR: 25.4 - 34.2) in the non-LIT group $(\mathrm{p}<0.001)$, parity with the median of 1 (IQR: $0-1)$ in the LIT group vs. 1 (IQR: 0 2 ) in the non-LIT group $(\mathrm{p}<0.001)$, and recipient MVP with the median of 11 (IQR: $9.3-13.3)$ in the LIT group vs. 10.1 (IQR $8.5-12.45)$ in the non-LIT group ( $\mathrm{p}=0.006)$ (Table 2).

\section{Operative variables}

Median GA at the time of FLS was 20.4 weeks which was similar between groups. The use of Solomon technique and CAS did not differ significantly between groups. Trocar outer sheath diameter was different between groups (median of $3.33 \mathrm{~mm}$ [IQR: $3.33-4$ ] in the LIT groupvs. $3.2 \mathrm{~mm}$ [IQR: $3.1-3.8$ ] in the non-LIT group, $\mathrm{p}<0.001$ ), total number of anastomoses were higher in the LIT group (median of 13 [IQR: $10-18$ ] vs. 11 [IQR: $8-13$ ], $\mathrm{p}<0.001$ ), and occurrence of postoperative septostomy was more common in the non-LIT group $(24.8 \%$ vs. $6.1 \%, \mathrm{p}<0.001)$ (Table 3$)$.

\section{Postoperative variables}

Gestational age at delivery was significantly different between the groups with median GA at delivery of 33.6 weeks (IQR: $31.2-35.3$ ) in the LIT groups vs. 31.1 weeks (IQR: $28.57-34)$ in the non-LIT group $(P$ $<0.001)$. PTB $<26$ weeks $(1.1 \%$ in the LIT group vs. $6.1 \%$ in the non-LIT group), extreme PTB $<28$ weeks ( $5.6 \%$ in the LIT group vs. $15.2 \%$ in the non-LIT group), PTB $<32$ weeks ( $27.8 \%$ in the LIT groupvs. $54.1 \%$ in the non-LIT), and PTB $<34$ weeks $(50.6 \%$ in the LIT group vs. $73.6 \%$ in the non-LIT) were all significantly higher in the non-LIT group $(\mathrm{p}=0.010, \mathrm{p}=0.002, \mathrm{p}<0.001$, and $\mathrm{p}<0.001$, respectively), Figure 2. FLS to delivery duration was significantly higher in the LIT group than in the non-LIT group with the median of 90 days (IQR: 70.35 - 108.85)vs. 76 days (IQR: $49-98)$, respectively $(\mathrm{p}<0.001)$ (Table 4$)$.

The risk of PTB prior to $34,32,28$, and 26 weeks was significantly lower in the LIT group compared to the non-LIT group $(\mathrm{RR}=0.69,0.51,0.37$, and 0.18 , respectively). Multivariate regression analysis showed race, BMI, parity, MVP, trocar outer sheath diameter, total number of anastomoses, and septostomy, are not associated with PTB while indomethacin use is a protective factor against PTB. (Table 5) The number needed to treat (NNT) with LIT to prevent one PTB $<32$ weeks gestation was 4 (95\% CI: 3.82 to 3.94), and to prevent one $\mathrm{PTB}<34$ weeks was 5 (95\% CI: 4.32 to 5.12 ).

Kaplan-Meier curve showed a significantly longer FLS-to-delivery duration in the LIT group compared to the non-LIT group (Log-rank test for equality of survival functions: $\mathrm{p}<0.001$, Figure 3 ). Multivariate stepwise backward Cox regression showed CL $<25 \mathrm{~mm}$, GA at intervention, LIT, and CAS were independent predictors of FLS-to-delivery survival. (Table 6) LIT remained a statistically significant predictor for longer 
FLS-to-delivery survival, even after adjusting for CL $<25 \mathrm{~mm}$, GA at intervention, and CAS (adjusted HR: $0.68,95 \%$ CI: $0.55-0.83)$.

There were no significant differences in live birth rates between the two groups. Neonatal survival $(>28$ days) of at least one newborn was increased in the LIT group compared to the non-LIT group (98.9\% vs. $95.7 \%)$, although this increase was not statistically significant $(\mathrm{p}=0.05)$. (Table 4 )

\section{LIT group characteristics}

A total of 180 pregnancies received LIT in the cohort (43.8\%). Median GA at the start of LIT was 20.4 weeks (IQR: $18.4-22.3$ ), and at the stop was 24.4 weeks (IQR: $22.2-26.6$ ), with a median duration of 21 days (IQR: 15.4 - 33.95). Reasons to stop LIT in descending order were: stable clinical status (149 cases, $83 \%$ ), 32 weeks gestation (13 cases, 7\%), PPROM (13 cases, 7\%), and fetal complications (4 cases, 2\%). Fetal complications included 3 fetuses $(1.5 \%)$ with constriction of DA and one fetus $(0.5 \%)$ with oligohydramnios (Table 7). All four cases of fetal complications were resolved after stopping LIT. The neonatal intensive care unit (NICU) chart review showed that the patient with resolved oligohydramnios fetus delivered at 30 weeks and the newborn had only prematurity-related respiratory distress syndrome (RDS). The three patients with resolved DA constriction fetuses were delivered at 32.4 weeks, 33.6 weeks, and 34.1 weeks. The first two newborns had RDS and bronchopulmonary dysplasia (BPD), while the third newborn had no complications. None of them required surgical intervention. All four were discharged home in stable condition. PPROM rate in the LIT cohort was $28.2 \%(\mathrm{n}=49)$ with a mean GA at PPROM of 29.6 weeks (IQR: $27.5-31.3)$. There was a moderate positive correlation, both between the duration of LIT and duration of FLS-to-PPROM $(\mathrm{r}=0.48, \mathrm{p}=0.002)$ and between the duration of LIT and duration of FLS-to-delivery $(\mathrm{r}=0.33, \mathrm{p}<0.001)$.

\section{Discussion}

This is the first study to evaluate the efficacy of LIT in prolonging pregnancy and reducing the risk of sPTB in the setting of TTTS treated with FLS. The main findings of the study were that the LIT group had a significantly longer pregnancy with a median of 2 weeks, longer FLS-to-delivery interval with a median of 2 weeks, $31 \%$ reduction in risk of PTB (GA less than 34 weeks), $52 \%$ reduction in risk of very PTB (GA less than 32 weeks), and $67 \%$ and $84 \%$ reduction in risk of extreme PTB (GA less than 28 and 26 weeks, respectively), and higher neonatal survival compared to the non-LIT group. Cox regression analysis showed that LIT is a significant predictor for prolongation of pregnancy following FLS, even after adjustment for CL, GA at FLS, and chorioamnion separation. The NNT with LIT to prevent one PTB $<32$ weeks gestation was 4 , and to prevent one $\mathrm{PTB}<34$ weeks was 5 .

PTB has been a major determinant for outcomes after FLS, with significant risk factors including PPROM, short preoperative CL, intraoperative amnioinfusion, and an increased number of anastomoses. ${ }^{7,18}$ Other reported risk factors include septostomy ${ }^{19-22}$ and chorioamnion separation. ${ }^{23-26}$ In our study, the multivariate regression analysis showed that none of these factors were significant independent predictors for PTB while LIT was the only significant independent protective factor in preventing PTB. Early gestational age at intervention ( $<18$ weeks), on the other hand, has not been found to increase the risk for PTB in this population $^{27}$, similarly to what our study showed.

The most common etiology for PTB is spontaneous PTB in 48 percent of patients. ${ }^{18}$ Effective PTB prevention measures in multiple gestations, in general, and in MCDA complicated with TTTS undergoing FLS, in specific, have not yet been defined. In multiple gestation with a normal or short cervix, interventions such as other forms of tocolytics, cerclage, progesterone, pessary, routine hospitalization, or bed rest have not been proven to prolong pregnancy or improve neonatal morbidity and mortality. ${ }^{10,28-40}$ In the setting of TTTS treated with FLS with short preoperative CL, cervical cerclage did not prolong pregnancy in a retrospective, multicenter cohort study. ${ }^{41}$ Our study showed that LIT use significantly increased the FLS-to-delivery interval regardless of the length of the cervix and gestational age of the surgery. 
The efficacy of short-term antenatal indomethacin use is well reported in the literature. In singleton pregnancies in the setting of preterm labor, a 2015 metanalysis of two randomized trials showed indomethacin compared to placebo was significantly effective in reducing the risk of delivery within 48 hours of initiation. ${ }^{42}$ In the setting of exam-indicated cerclage, indomethacin along with antibiotics significantly prolonged pregnancy for more than 28 days. ${ }^{43}$ In twin pregnancies, a retrospective matched case-control study previously reported that LIT stabilizes cervical length in DCDA twin gestations with short CL. ${ }^{12}$ It is important to emphasize that our study demonstrated that LIT reduces the risk of extreme prematurity by $67 \%$ and $84 \%$ (GA less than 28 and 26 weeks, respectively).

PPROM has been reported to be as high as $53 \%$ after FLS. ${ }^{5,8,9}$ In PPROM generally, sequence of events modulates metalloproteinases (MMPs) expression, secretion, and activation by local and infiltrating cells synchronously with myometrial and cervical changes. This leads to the progressive loss of the mechanical properties and tensile strength of the amniotic membranes and eventually to their rupture. ${ }^{44-49}$ Similar cascade of events has been shown in amniotic membranes following FLS. ${ }^{50}$ In our study, longer duration of LIT use was associated with longer FLS-to-delivery interval. This effect of LIT has significant rationale given that indomethacin has multiple vectors of impact, including tocolysis, uterine relaxation, biochemical cervical stabilization, and hydrophobic effect on MMPs. ${ }^{11}$

In terms of indomethacin safety, data on fetal effects have been conflicting, with the main concerns being oligohydramnios and constriction of ductus arteriosus, both of which have been shown to resolve within 48 hours of stopping the medication. ${ }^{51,52}$ Neonatal effects associated with antenatal use of indomethacin have also been controversial, mostly related to the prematurity associated adverse events aside from the use of indomethacin. In a meta-analysis including randomized trials, there were no statistically significant differences in adverse events between cases with indomethacin use and controls ${ }^{53}$ while a systematic review of case-control pairs that were not matched for GA or birth weight showed an increase in neonatal morbidity. ${ }^{54}$ Other reported fetal and neonatal outcome data are mainly in the form of case reports and case series. ${ }^{55-62}$ Turan et al. investigated the safety of LIT in a matched case-control analysis considering the effects of GA and birth weight on neonatal morbidities. That study showed no significant increase in neither fetal nor neonatal complications in cases underwent LIT. ${ }^{13}$ In the current study, there were only four indomethacinrelated fetal complications, including one oligohydramnios and three DA constrictions. All four resolved after stopping LIT, and all four newborns were discharged home in stable condition.

Our study has several strengths. To the best of our knowledge, this is the first study reporting on the effect of LIT in prolonging pregnancy and reducing the risk of SPTB in monochorionic pregnancies complicated by TTTS that required FLS. The study was conducted in two experienced, high volume fetal centers. The data were collected from all consecutive patients who underwent FLS which minimizes the selection bias. The data were collected prospectively as an ongoing process, which reduces recollection bias and missing data. The establishment of a vigorous, standardized LIT protocol allowed for uniformly proper follow-up of these patients. The sample size was large enough to assess the effect of the most relevant risk factors in a regression model.

We acknowledge the limitations of our study, including the retrospective nature of the study. The study was conducted in two different centers; therefore, different institutional practices or techniques might have impacted the outcomes despite following similar procedural indications and follow-up protocols between the two participating institutions. Other limitations include the lack of neonatal outcomes comparisons between the groups aside from perinatal survival.

In conclusion, in the setting of TTTS treated with FLS, LIT; defined as indomethacin use for more than 48 hours and not beyond 32 weeks gestation; was significantly effective in prolonging pregnancy with a median of 2 weeks and reducing the risk of PTB particularly the risk of extreme prematurity, which has the highest impact on neonatal outcomes. Future prospective randomized trials are required to confirm our findings and investigate the effect of LIT in other clinical settings such as in patients with short cervical length. 


\section{Disclosure of interests}

None declared. Completed disclosure of interests forms are available to view online as supporting information.

\section{Contribution to authorship}

HJM and OMT conceived the study and designed the protocol. HJM and NP collected the data. HJM wrote the first draft of the paper. OMT and HT were involved in the statistical analysis and drafting the results. EK, JE, AS, AN, RD, MB, MC, and $\mathrm{CH}$ were all involved in critical analysis of the data. All authors contributed significantly to critical revisions of successive drafts of the manuscript. HJM and OMT are the guarantors of the review.

\section{Funding}

None.

\section{Acknowledgements}

The authors would like to thank all the staff and workers of the fetal care center at the University of Maryland and Baylor college of Medicine for their hard work and dedication to deliver the best care for our patients.

\section{References}

1 NJ, S., RJ, S., K, H., W, S. \& KH, N. The hidden mortality of monochorionic twin pregnancies. British journal of obstetrics and gynaecology 104, doi:10.1111/j.1471-0528.1997.tb10948.x (1997).

$2 \mathrm{MV}$, S. et al. Endoscopic laser surgery versus serial amnioreduction for severe twin-to-twin transfusion syndrome. The New England journal of medicine 351, doi:10.1056/NEJMoa032597 (2004).

3 R, L. et al. Neonatal outcome in preterm monochorionic twins with twin-to-twin transfusion syndrome after intrauterine treatment with amnioreduction or fetoscopic laser surgery: comparison with dichorionic twins. American journal of obstetrics and gynecology 196 , doi:10.1016/j.ajog.2007.01.036 (2007).

4 R, R. et al. Cervical length as a prognostic factor for preterm delivery in twin-to-twin transfusion syndrome treated by fetoscopic laser coagulation of chorionic plate anastomoses. Ultrasound in obstetrics $\mathbb{E}$ gynecology : the official journal of the International Society of Ultrasound in Obstetrics and Gynecology 25 , doi:10.1002/uog.1798 (2005).

5 V, B., P, L., L, G. \& R, D. Preterm prelabor rupture of membranes and fetal survival after minimally invasive fetal surgery: a systematic review of the literature. Fetal diagnosis and therapy31, doi:10.1159/000331165 (2012).

$6 \mathrm{M}$, H. et al. Incidence of complications in twin-twin transfusion syndrome after selective fetoscopic laser photocoagulation: a single-center experience. American journal of obstetrics and gynecology 201 , doi:10.1016/j.ajog.2009.07.046 (2009).

7 Ramesha PAPANNA, M. D., M.P.H.,1,2 Dana BLOCK-ABRAHAM, D.O.,4 Lovepreet K Mann, M.B.B.S.,3 Irina A. BUHIMSCHI, M.D., M.M.S.,1 Michael BEBBINGTON, M.D., M.H.Sc.,3 Elisa GARCIA, B.A.,3 Nahla KAHLEK, M.D.,5 Christopher HARMAN, M.D.,4 Anthony JOHNSON, D.O.,3 Ahmet BASCHAT, M.D.,4 and Kenneth J. MOISE, Jr., M.D.3.

8 Stirnemann, J. et al. Preterm premature rupture of membranes is a collateral effect of improvement in perinatal outcomes following fetoscopic coagulation of chorionic vessels for twin-twin transfusion syndrome: a retrospective observational study of 1092 cases. BJOG125 , 1154-1162, doi:10.1111/1471-0528.15147 (2018). 
9 Snowise, S. et al. Preterm prelabor rupture of membranes after fetoscopic laser surgery for twin-twin transfusion syndrome. Ultrasound Obstet Gynecol 49, 607-611, doi:10.1002/uog.15958 (2017).

10 CN, L., P, Z. \& RC, Y. Differing mechanisms of inhibition of calcium increases in human uterine myocytes by indomethacin and nimesulide.American journal of obstetrics and gynecology $\mathbf{1 8 4}$, doi:10.1067/mob.2001.114915 (2001).

11 J, K.-B. et al. Effect of indometacin pretreatment on protamine sulfate-mediated relaxation of the isolated rat uterus: the role of the antioxidative defense system. Pharmacological reports : PR 63, doi:10.1016/s17341140(11)70618-1 (2011).

12 Shannon, A. The Use of Long-Term Indomethacin Stabilizes Cervical Length... : Obstetrics \& Gynecology. doi:10.1097/01.AOG.0000514117.88934.6d (2021).

13 Turan, O. M. Prolonged early antenatal indomethacin exposure is safe for fetus and neonate.

14 von Elm, E. et al. The Strengthening the Reporting of Observational Studies in Epidemiology (STROBE) statement: guidelines for reporting observational studies. The Lancet 370 , 1453-1457, doi:10.1016/S01406736(07)61602-X (2007).

15 RA, Q. et al. Staging of twin-twin transfusion syndrome. Journal of perinatology : official journal of the California Perinatal Association 19, doi:10.1038/sj.jp.7200292 (1999).

$16 \mathrm{~F}$, S. et al. Fetoscopic laser coagulation of the vascular equator versus selective coagulation for twinto-twin transfusion syndrome: an open-label randomised controlled trial. Lancet (London, England) 383 , doi:10.1016/S0140-6736(13)62419-8 (2014).

17 Stata Statistical Software v. Release 16 (StataCorp LLC, College Station, TX, 2019).

18 Malshe, A. Preterm delivery after fetoscopic laser surgery for twin-twin transfusion syndrome: etiology and risk factors - Malshe - 2017 - Ultrasound in Obstetrics \& Gynecology - Wiley Online Library. doi:10.1002/uog.15972 (2021).

19 R, C.-M. et al. Incidence and clinical implications of early inadvertent septostomy after laser therapy for twin-twin transfusion syndrome. Ultrasound in obstetrics 85 gynecology : the official journal of the International Society of Ultrasound in Obstetrics and Gynecology 37, doi:10.1002/uog.8922 (2011).

$20 \mathrm{SH}, \mathrm{P}$. et al. Iatrogenic perforation of intertwin membrane after laser surgery for twin-to-twin transfusion syndrome.Ultrasound in obstetrics \& gynecology : the official journal of the International Society of Ultrasound in Obstetrics and Gynecology44, doi:10.1002/uog.13445 (2014).

$21 \mathrm{WF}$, L. et al. Effects and outcomes of septostomy in twin-to-twin transfusion syndrome after fetoscopic laser therapy.BMC pregnancy and childbirth 19, doi:10.1186/s12884-019-2555-5 (2019).

22 AA, N. et al. Perinatal outcome of twin-to-twin transfusion syndrome complicated with incidental septostomy after laser surgery: a systematic review and meta-analysis. Prenatal diagnosis, doi:10.1002/pd.5935 (2021).

23 JU, O. et al. Chorioamniotic membrane separation after fetoscopy in monochorionic twin pregnancy: incidence and impact on perinatal outcome. Ultrasound in obstetrics $\& 5$ gynecology : the official journal of the International Society of Ultrasound in Obstetrics and Gynecology 47, doi:10.1002/uog.14936 (2016).

24 M, T., M, N., S, M., M, S. \& M, M. Chorioamniotic Membrane Separation after Fetoscopic Laser Photocoagulation. Fetal diagnosis and therapy 43 , doi:10.1159/000472713 (2018).

25 R, P., LK, M., A, J., H, S.-H. \& KJ, M. Chorioamnion separation as a risk for preterm premature rupture of membranes after laser therapy for twin-twin transfusion syndrome. Obstetrics and gynecology $\mathbf{1 1 5}$ , doi:10.1097/AOG.0b013e3181d57335 (2010). 
26 AA, N. et al. Perinatal outcomes of iatrogenic chorioamniotic separation following fetoscopic surgeries: a systematic review and meta-analysis. Ultrasound in obstetrics $\&$ gynecology : the official journal of the International Society of Ultrasound in Obstetrics and Gynecology, doi:10.1002/uog.23588 (2021).

27 Espinoza, J. et al. Early Laser Surgery is not associated with very Preterm Delivery or Reduced Neonatal Survival in TTTS. Ultrasound Obstet Gynecol, doi:10.1002/uog.22190 (2020).

28 A, R. et al. Physical examination-indicated cerclage in twin pregnancy: a randomized controlled trial. American journal of obstetrics and gynecology 223, doi:10.1016/j.ajog.2020.06.047 (2020).

29 CA, C. \& S, H. Hospitalisation and bed rest for multiple pregnancy. The Cochrane database of systematic reviews 2010, doi:10.1002/14651858.CD000110.pub2 (2010).

30 CA, C., T, G., K, M., A, D. \& M, P. 17-hydroxyprogesterone caproate for twin pregnancy: a double-blind, randomized clinical trial.American journal of obstetrics and gynecology 204, doi:10.1016/j.ajog.2010.12.042 (2011).

31 CL, C. \& RK, F. Ritodrine HCL for the prevention of premature labor in twin pregnancies. Acta geneticae medicae et gemellologiae $\mathbf{2 5}$, doi:10.1017/s0001566000014355 (1976).

32 DJ, R. et al. A trial of 17 alpha-hydroxyprogesterone caproate to prevent prematurity in twins. The New England journal of medicine $\mathbf{3 5 7}$, doi:10.1056/NEJMoa070641 (2007).

33 EB, F., E, C., M, P., M, S. \& KH, N. Progesterone and the risk of preterm birth among women with a short cervix. The New England journal of medicine 357, doi:10.1056/NEJMoa067815 (2007).

$34 \mathrm{JE}, \mathrm{N}$. et al. Progesterone for the prevention of preterm birth in twin pregnancy (STOPPIT): a randomised, double-blind, placebo-controlled study and meta-analysis. Lancet (London, England) 373 , doi:10.1016/S0140-6736(09)60947-8 (2009).

$35 \mathrm{MV}$, S. et al. Prevention of preterm delivery by 17 alpha-hydroxyprogesterone caproate in asymptomatic twin pregnancies with a short cervix: a randomized controlled trial. American journal of obstetrics and gynecology 208 , doi:10.1016/j.ajog.2013.01.032 (2013).

$36 \mathrm{~S}, \mathrm{~L}$. et al. Cervical pessaries for prevention of preterm birth in women with a multiple pregnancy (ProTWIN): a multicentre, open-label randomised controlled trial. Lancet (London, England)382 , doi:10.1016/S0140-6736(13)61408-7 (2013).

$37 \mathrm{~S}, \mathrm{~W}$. et al. Vaginal progesterone to prevent preterm birth in multiple pregnancy: a randomized controlled trial. Journal of perinatal medicine 40 , doi:10.1515/jpm-2012-0057 (2012).

38 SN, C. et al. Prevention of preterm birth in triplets using 17 alpha-hydroxyprogesterone caproate: a randomized controlled trial.Obstetrics and gynecology 113, doi:10.1097/AOG.0b013e318193c677 (2009).

39 C, R. et al. Arabin cervical pessary for prevention of preterm birth in cases of twin-to-twin transfusion syndrome treated by fetoscopic LASER coagulation: the PECEP LASER randomised controlled trial. BMC pregnancy and childbirth 17, doi:10.1186/s12884-017-1435-0 (2017).

40 MV, Z. et al. North American Fetal Therapy Network: Timing of and indications for delivery following laser ablation for twin-twin transfusion syndrome. American journal of obstetrics $\&$ gynecology MFM $\mathbf{1}$, doi:10.1016/j.ajogmf.2019.02.001 (2019).

41 Papanna, R. et al. Cerclage for cervical shortening at fetoscopic laser photocoagulation in twin-twin transfusion syndrome.Am J Obstet Gynecol 206 , 425 e421-427, doi:10.1016/j.ajog.2012.02.022 (2012).

$42 \mathrm{HE}$, R. et al. Cyclo-oxygenase (COX) inhibitors for treating preterm labour. The Cochrane database of systematic reviews2015, doi:10.1002/14651858.CD001992.pub3 (2015).

43 ES, M., WA, G., L, F. \& BK, R. Indomethacin and antibiotics in examination-indicated cerclage: a randomized controlled trial.Obstetrics and gynecology 123, doi:10.1097/AOG.0000000000000228 (2014). 
44 SJ, F., R, M., C, B. \& SJ, L. Programmed cell death (apoptosis) as a possible pathway to metalloproteinase activation and fetal membrane degradation in premature rupture of membranes. American journal of obstetrics and gynecology $\mathbf{1 8 2}$, doi:10.1067/mob.2000.107330 (2000).

45 S, P. \& JF, S. Premature rupture of the fetal membranes. The New England journal of medicine 338 , doi:10.1056/NEJM199803053381006 (1998).

$46 \mathrm{~N}$, A. et al. A role for matrix metalloproteinase-9 in spontaneous rupture of the fetal membranes. American journal of obstetrics and gynecology 179, doi:10.1016/s0002-9378(98)70141-3 (1998).

47 J, M., DJ, T. \& SC, B. Increased concentration of pro-matrix metalloproteinase 9 in term fetal membranes overlying the cervix before labor: implications for membrane remodeling and rupture. American journal of obstetrics and gynecology 182 , doi:10.1016/s0002-9378(00)70232-8 (2000).

48 Estrada-Gutierrez, F. V. O. G. Role of matrix metalloproteinases in preterm labour - Vadillo-Ortega - 2005 - BJOG: An International Journal of Obstetrics \& Gynaecology - Wiley Online Library. doi:10.1111/j.14710528.2005.00579.x (2021).

49 JF, S. Extracellular matrix dynamics and fetal membrane rupture.Reproductive sciences (Thousand Oaks, Calif.) 20 , doi:10.1177/1933719111424454 (2013).

50 Papanna. Histologic changes of the fetal membranes after fetoscopic laser surgery for twin-twin transfusion syndrome | Pediatric Research , <https://www.nature.com/articles/pr2015105> (2015).

51 JC, S., WA, G. \& SE, G. The effect of short-term indomethacin therapy on amniotic fluid volume. American journal of obstetrics and gynecology 192, doi:10.1016/j.ajog.2004.12.036 (2005).

52 RJ, S., S, L., NM, F. \& PR, B. A double-blind randomized study of fetal side effects during and after the short-term maternal administration of indomethacin, sulindac, and nimesulide for the treatment of preterm labor. American journal of obstetrics and gynecology 188, doi:10.1067/mob.2003.255 (2003).

53 SM, L., L, S.-R. \& AM, K. Assessing the neonatal safety of indomethacin tocolysis: a systematic review with meta-analysis. Obstetrics and gynecology 106 , doi:10.1097/01.AOG.0000168622.56478.df (2005).

54 AL, H., L, S.-R. \& AM, K. Antenatal exposure to indomethacin increases the risk of severe intraventricular hemorrhage, necrotizing enterocolitis, and periventricular leukomalacia: a systematic review with metaanalysis. American journal of obstetrics and gynecology212, doi:10.1016/j.ajog.2014.10.1091 (2015).

$55 \mathrm{~A}$, P. et al. Acute renal failure in the neonate induced by the administration of indomethacin as a tocolytic agent. Nephrology, dialysis, transplantation : official publication of the European Dialysis and Transplant Association - European Renal Association 11 (1996).

56 AS, S., S, D. \& N, S. ACOG_Antenatal indomethacin tocolysis is associated with an increased need for surgical ligation of patent ductus arteriosus in preterm infants. Journal of obstetrics and gynaecology Canada : JOGC = Journal d'obstetrique et gynecologie du Canada $:$ JOGC 32, doi:10.1016/S1701-2163(16)34496-6 (2010).

57 B, K., KJ, M., G, M. \& R, W. 6 fetuses_Long-term indomethacin therapy decreases fetal urine output and results in oligohydramnios. American journal of perinatology 8 , doi:10.1055/s-2007-999349 (1991).

58 BG, S. et al. ACOG_The risk of necrotizing enterocolitis after indomethacin tocolysis. Pediatrics 128 , doi:10.1542/peds.2011-0265 (2011).

59 BV, A. P., WA, G., RB, H., HA, T. \& SL, D. ACOG_Indomethacin tocolysis and risk of necrotizing enterocolitis. Obstetrics and gynecology 96 , doi:10.1016/s0029-7844(00)00846-2 (2000).

60 S, A. A., JS, G., HM, S., SS, S. \& J, L. Neonatal outcome after exposure to indomethacin in utero: a retrospective case cohort study.American journal of obstetrics and gynecology 189, doi:10.1067/s00029378(03)00662-8 (2003). 
61 SK, H., JR, S., DE, M. \& ZA, B. Oligohydramnios associated with prostaglandin synthetase inhibitors in preterm labour. British journal of obstetrics and gynaecology $\mathbf{9 7}$, doi:10.1111/j.1471-0528.1990.tb01807.x (1990).

$62 \mathrm{~T}$, N. et al. Renal impairment in very low birthweight infants following antenatal indomethacin administration. Acta paediatrica Japonica : Overseas edition 36, doi:10.1111/j.1442-200x.1994.tb03162.x (1994).

\section{Tables}

Table 1. The distribution of excluded pregnancies by centers

\begin{tabular}{|c|c|c|c|}
\hline Exclusion criteria & UMB N (262) & BCM N (295) & Total N (557) \\
\hline Triplet gestation & $16(6.1 \%)$ & $17(5.8 \%)$ & $33(5.9 \%)$ \\
\hline Cerclage placement & $9(3.4 \%)$ & $13(4.4 \%)$ & $22(3.9 \%)$ \\
\hline Dual twin demise & $8(3.0 \%)$ & $10(3.4 \%)$ & $18(3.2 \%)$ \\
\hline $\begin{array}{l}\text { Indicated delivery }<32 \\
\text { weeks }\end{array}$ & $20(7.6 \%)$ & $19(7.2 \%)$ & $39(7 \%)$ \\
\hline Missing data & $18(6.8 \%)$ & $16(5.4 \%)$ & $34(6.1 \%)$ \\
\hline Total $n$ & $71(27 \%)$ & $75(25.4 \%)$ & $146(26.2 \%)$ \\
\hline Data are given as n (\%) & Data are given as n $(\%)$ & Data are given as $\mathrm{n}(\%)$ & Data are given as n (\%) \\
\hline UMB: University of & UMB: University of & UMB: University of & UMB: University of \\
\hline Maryland, Baltimore; & Maryland, Baltimore; & Maryland, Baltimore; & Maryland, Baltimore; \\
\hline BCM: Baylor College & BCM: Baylor College & BCM: Baylor College & BCM: Baylor College \\
\hline of Medicine & of Medicine & of Medicine & of Medicine \\
\hline
\end{tabular}

Table 2. Maternal characteristics and preoperative variables comparisons between the groups

\begin{tabular}{llll}
\hline Preoperative Variable & LIT N (180) & Non-LIT N (231) & P value \\
\hline $\begin{array}{l}\text { Maternal age (years) } \\
\text { Parity }\end{array}$ & $30(25.5 .-34.5)$ & $29(25-33)$ & 0.07 \\
Race, $n$ (\%) & $1(0-1)$ & $1(0-2)$ & $<0.001$ \\
White & $124(69.3 \%)$ & $126(54.5 \%)$ & $<0.001$ \\
Black & $24(13.4 \%)$ & $22(9.5 \%)$ & \\
Hispanic & $6(3.4 \%)$ & $72(31.2 \%)$ & \\
Other & $25(14.0 \%)$ & $11(4.8 \%)$ & \\
Prior PTB, $n(\%)$ & $17(9.8 \%)$ & $31(13.4 \%)$ & 0.27 \\
BMI $\left(\mathrm{kg} / \mathrm{m}^{2}\right)$ & $23.9(21-29.4)$ & $28.2(25.4-34.2)$ & $<0.001$ \\
sFGR, $n(\%)$ & $80(47.1 \%)$ & $103(45.0 \%)$ & 0.68 \\
CL $<25 \mathrm{~mm}, n(\%)$ & $11(6.1 \%)$ & $21(9.4 \%)$ & 0.22 \\
Anterior placenta, $n$ & $71(39.4 \%)$ & $102(44.2 \%)$ & 0.34 \\
(\%) & & & \\
Recipient MVP $(\mathrm{cm})$ & $10.1(8.5-12.45)$ & $11(9.3-13.3)$ & 0.006 \\
Quintero staging, $n$ & & & 0.16 \\
(\%) & $32(17.8 \%)$ & $27(11.7 \%)$ & \\
I & $62(34.4 \%)$ & $70(30.3 \%)$ & \\
II & $75(41.7 \%)$ & $118(51.1 \%)$ & \\
III & $11(6.1 \%)$ & $16(6.9 \%)$ & \\
IV & & &
\end{tabular}




\begin{tabular}{llll}
\hline Preoperative Variable & LIT N (180) & Non-LIT N (231) & P value \\
\hline Data are given as & Data are given as & Data are given as & Data are given as \\
median (IQR) and n & median (IQR) and n & median (IQR) and n & median (IQR) and n \\
(\%) PTB: preterm & (\%) PTB: preterm & (\%) PTB: preterm & (\%) PTB: preterm \\
birth, BMI: body mass & birth, BMI: body mass & birth, BMI: body mass & birth, BMI: body mass \\
index, sFGR: selective & index, sFGR: selective & index, sFGR: selective & index, sFGR: selective \\
fetal growth restriction, & fetal growth restriction, & fetal growth restriction, & fetal growth restriction, \\
CL: cervical length, & CL: cervical length, & CL: cervical length, & CL: cervical length, \\
MVP: maximum & MVP: maximum & MVP: maximum & MVP: maximum \\
vertical pocket & vertical pocket & vertical pocket & vertical pocket \\
\hline
\end{tabular}

Table 3 . Operative variables comparisons between the groups

\begin{tabular}{|c|c|c|c|}
\hline Operative Variable & LIT N (180) & Non-LIT N (231) & $\mathrm{P}$ value \\
\hline GA at FLS (weeks) & $20.4(18.4-22.3)$ & $20.4(18.5-22.57)$ & 0.53 \\
\hline $\begin{array}{l}\text { GA at } \mathrm{FLS}<18 \\
\text { (weeks) }\end{array}$ & $31(17.2 \%)$ & $41(17.7 \%)$ & 0.89 \\
\hline $\begin{array}{l}\text { Trocar outer sheath } \\
\text { diameter }(\mathrm{mm})\end{array}$ & $3.2(3.1-3.8)$ & $3.33(3.33-4)$ & $<0.001$ \\
\hline Number of anastomoses & $13(10-18)$ & $11(8-13)$ & $<0.001$ \\
\hline $\begin{array}{l}\text { Solomon technique, } n \\
\text { (\%) }\end{array}$ & $129(74.6 \%)$ & $181(80.1 \%)$ & 0.19 \\
\hline Septostomy, $n$ (\%) & $11(6.1 \%)$ & $57(24.8 \%)$ & $<0.001$ \\
\hline $\mathrm{CAS}, n(\%)$ & $14(7.8 \%)$ & $31(13.7 \%)$ & 0.60 \\
\hline $\begin{array}{l}\text { Data are given as } \\
\text { median (IQR) and n } \\
\text { (\%) GA: gestational } \\
\text { age, FLS: fetoscopic } \\
\text { laser surgery, CAS: } \\
\text { chorioamnion } \\
\text { separation }\end{array}$ & $\begin{array}{l}\text { Data are given as } \\
\text { median (IQR) and n } \\
\text { (\%) GA: gestational } \\
\text { age, FLS: fetoscopic } \\
\text { laser surgery, CAS: } \\
\text { chorioamnion } \\
\text { separation }\end{array}$ & $\begin{array}{l}\text { Data are given as } \\
\text { median (IQR) and n } \\
\text { (\%) GA: gestational } \\
\text { age, FLS: fetoscopic } \\
\text { laser surgery, CAS: } \\
\text { chorioamnion } \\
\text { separation }\end{array}$ & $\begin{array}{l}\text { Data are given as } \\
\text { median (IQR) and n } \\
\text { (\%) GA: gestational } \\
\text { age, FLS: fetoscopic } \\
\text { laser surgery, CAS: } \\
\text { chorioamnion } \\
\text { separation }\end{array}$ \\
\hline
\end{tabular}

Table 4 . Postoperative variables comparisons between the groups

\begin{tabular}{llll}
\hline Postoperative Variable & LIT N (180) & Non-LIT N $(231)$ & P value \\
\hline $\begin{array}{l}\text { GA at delivery (weeks) } \\
\text { GA at delivery }<26\end{array}$ & $33.6(31.2-35.3)$ & $31.1(28.57-34)$ & $<0.001$ \\
$\begin{array}{l}\text { weeks, } n \text { (\%) } \\
\text { GA at delivery }<28\end{array}$ & $10(5.6 \%)$ & $14(6.1 \%)$ & 0.01 \\
$\begin{array}{l}\text { weeks, } n(\%) \\
\text { GA at delivery }<32\end{array}$ & $50(27.8 \%)$ & $35(15.2 \%)$ & 0.002 \\
weeks, $n(\%)$ & $125(54.1 \%)$ & $<0.001$ \\
GA at delivery $<34$ & $91(50.6 \%)$ & $170(73.6 \%)$ & $<0.001$ \\
weeks, $n(\%)$ & $76(49-98)$ & $<0.001$ \\
$\begin{array}{l}\text { FLS-to-delivery (days) } \\
\text { At least one live birth, } \\
n(\%)\end{array}$ & $90(70.35-108.85)$ & $231(100 \%)$ & 0.26
\end{tabular}




\begin{tabular}{|c|c|c|c|}
\hline Postoperative Variable & LIT N (180) & Non-LIT N (231) & $\mathrm{P}$ value \\
\hline $\begin{array}{l}\text { At least one neonatal } \\
\text { survival ( }>28 \text { days), } n \\
\text { (\%) }\end{array}$ & $178(98.9 \%)$ & $221(95.7 \%)$ & 0.05 \\
\hline $\begin{array}{l}\text { Data are given as } \\
\text { median (IQR) and n } \\
\text { (\%) GA: gestational } \\
\text { age, FLS: fetoscopic } \\
\text { laser surgery }\end{array}$ & $\begin{array}{l}\text { Data are given as } \\
\text { median (IQR) and n } \\
\text { (\%) GA: gestational } \\
\text { age, FLS: fetoscopic } \\
\text { laser surgery }\end{array}$ & $\begin{array}{l}\text { Data are given as } \\
\text { median (IQR) and n } \\
\text { (\%) GA: gestational } \\
\text { age, FLS: fetoscopic } \\
\text { laser surgery }\end{array}$ & $\begin{array}{l}\text { Data are given as } \\
\text { median (IQR) and n } \\
\text { (\%) GA: gestational } \\
\text { age, FLS: fetoscopic } \\
\text { laser surgery }\end{array}$ \\
\hline
\end{tabular}

Table 5. Relative risk for PTB in the LIT group vs. non-LIT group

\begin{tabular}{|c|c|}
\hline Variable & GA $<26$ weeks \\
\hline & Univariate model \\
\hline & RR $(95 \% \mathrm{CI})$ \\
\hline LIT & $0.18(0.04-0.8)$ \\
\hline \multicolumn{2}{|l|}{ Parity } \\
\hline 0 & Ref. \\
\hline 1 & $0.8(0.23-2.76)$ \\
\hline 2 & $1.52(0.5-4.58)$ \\
\hline \multicolumn{2}{|l|}{ Race } \\
\hline White & Ref. \\
\hline Black & - \\
\hline Hispanic & $1.92(0.72-5.12)$ \\
\hline Other & - \\
\hline \multicolumn{2}{|l|}{ BMI } \\
\hline Normal & Ref. \\
\hline Overweight & $1.06(0.35-3.21)$ \\
\hline Obese & $0.68(0.2-2.34)$ \\
\hline \multicolumn{2}{|l|}{ Recipient MVP } \\
\hline Normal & Ref. \\
\hline Polyhydramnios & $0.76(0.1-6.12)$ \\
\hline Severe polyhydramnios & $1.86(0.24-14.15)$ \\
\hline \multicolumn{2}{|l|}{ Trocar outer shea } \\
\hline$<3.1$ & Ref. \\
\hline $3.1-3.33$ & $0.48(0.12-1.89)$ \\
\hline $3.33-3.8$ & $0.44(0.05-3.56)$ \\
\hline 3.8 & $1.45(0.48-4.36)$ \\
\hline \multicolumn{2}{|l|}{ Anastomoses } \\
\hline$<9$ & Ref. \\
\hline $9-12$ & $1.19(0.33-4.28)$ \\
\hline $12-15$ & $0.75(0.17-3.26)$ \\
\hline 15 & $0.8(0.21-3.12)$ \\
\hline Septostomy & $1.16(0.34-3.96)$ \\
\hline RR: relative risk; CI: confidence interval EXPLAIN OTHER ABBREVIATIONS & RR: relative risk; CI: confidence interval \\
\hline
\end{tabular}

Table 6. Hazard ratio for FLS-to-delivery survival 
Variable

LIT

$\mathrm{CL}<25 \mathrm{~mm}$

Chorioamnion separation

GA at FLS (weeks)

LIT: long-term indomethacin therapy, CL: cervical length, GA: gestational age, FLS: fetal laser surgery HR: hazard ratio, C

Table 7. Characteristics of the LIT group

LIT Group Characteristics, $\mathrm{n}=180$

GA at LIT start (weeks)

GA at LIT stop (weeks)

Reasons LIT stopped, $n(\%)$

Stable clinical status *

32 weeks

PPROM

Fetal complications DA constriction Oligohydramnios

LIT duration (days)

Data are given as median (IQR) and n (\%) *The definition can be seen in the methods section under LIT protocol GA: ges

\section{Figures legends}

Figure 1. Flow diagram of study population

Figure 2. The rate of preterm births before 26, 28, 32, and 34 weeks gestation by long-term indomethacin (LIT) and non-LIT groups

Figure 3. The probability of staying pregnant after fetoscopic laser surgery (FLS) by long-term indomethacin (LIT) and non-LIT groups 


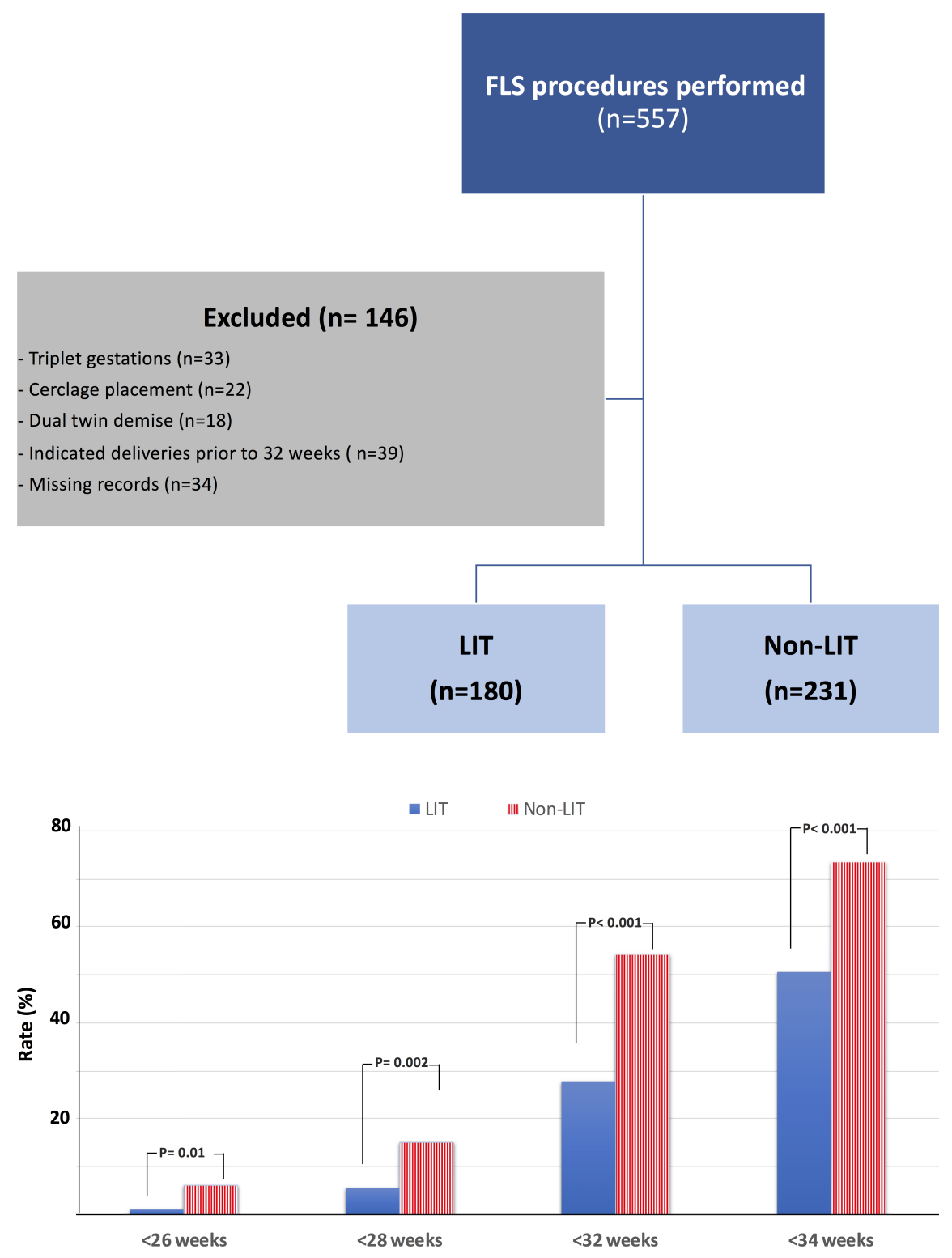




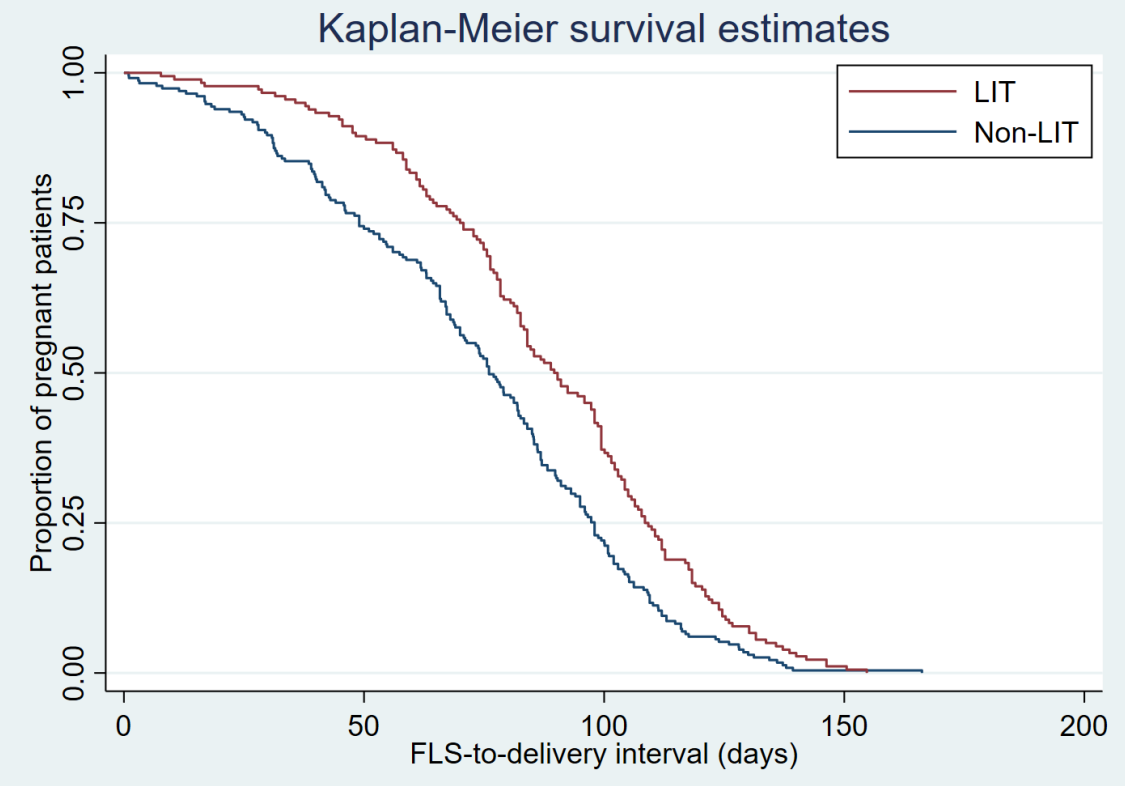

